\title{
SINGULARITY ANALYSIS THROUGH STATIC ANALYSIS
}

\author{
J. Hubert \\ INRIA Sophia-Antipolis \\ Julien.Hubert@inria.fr \\ J-P. Merlet \\ INRIA Sophia-Antipolis \\ Jean-Pierre.Merlet@inria.fr
}

Abstract bla bla bla abstract.

Keywords: force workspace, planar parallel robots, singularity

\section{Introduction}

The singularities of parallel robots have been studied since the 1980s. According to the authors knowledge, the first study concerning singular configurations was done by Hunt[] who defined stationary and uncertainty configurations.

In particular poses, the end-effector is not able to follow certain directions that it is normaly able to. That is why it is said that in stationary configurations the mechanism loses some of its degrees of freedom.

On the other side, uncertainty configurations correspond to the endeffector inabilityy to resist to a given wrench, so the robot gains degrees of freedom. This condition may be found with kinematic or static analysis as in [].

One very interesting singularity phenomenon revealed by static analysis is the existence of a load such that in the neighbourhood of a singularity, the internal forces in the joints of the structure tends to infinity. Obviously such large forces have led to the breakdown of some research and industrial prototypes.

So considering the designer's point of view, a very useful information to build a robot is the singularity loci. Unfortunately this is not sufficient because the value of the force that can be applied on a leg is limited by the strength of the materila as well as the cost constraint.

However, if the load is known, it exists areas where the internal forces in the joint structures $\tau$ are such that $\tau \leq \tau_{\max }$ where $\tau_{\max }$ is a threshold defined by the designer. Note that these regions may contain singulari- 
ties on their border. These areas defined the force workspace which will be more useful to designer than the singularity loci. So we extend the concept of singularity initially associated with points where the determinent of the Jacobian is equal to zero to a region around these points where it is certain that the internal forces are greater than $\tau_{\max }$.

The purpose of this paper is to present an algorithm which allow us to compute the force workspace with a given orientation of the endeffector, a given load and $\tau_{\max }$ for the well known planar parallel robot $3-R \underline{P} R$.

\section{Static Analysis}

We will follow the notations as given in [].

At static equilibrium, the fundamental relation between the joint forces vector $\tau$, the external wrench exerted on the environment $\mathrm{F}$ and the kinematic Jacobian matrix $\mathbf{J}$ is given by :

$$
\mathcal{F}=\mathbf{J}^{-T} \tau
$$

We recall that a line $J_{i}$ of $J$ is given by

$$
J_{i}=\left(\frac{\mathbf{A}_{\mathbf{i}} \mathbf{B}_{\mathbf{i}}}{\rho_{i}} \quad \frac{\mathbf{C B}_{\mathbf{i}} \times \mathbf{A}_{\mathbf{i}} \mathbf{B}_{\mathbf{i}}}{\rho_{i}}\right)
$$

where $\rho_{i}=|| \mathbf{A}_{\mathbf{i}} \mathbf{B}_{\mathbf{i}} \|$

A matrix $\mathbf{M}$ can then be constructed such that its columns $M_{i}$ are defined as :

$$
M_{i}=\left(\begin{array}{ll}
\mathbf{A}_{\mathbf{i}} \mathbf{B}_{\mathbf{i}} & \mathbf{C B}_{\mathbf{i}} \times \mathbf{A}_{\mathbf{i}} \mathbf{B}_{\mathbf{i}}
\end{array}\right)^{T}
$$

and the matrices $\mathbf{N}_{\mathbf{i}}$ that are obtained by the substitution of the i-th column of $\mathbf{J}^{T}$ by $\mathcal{F}$

According to the Cramer's rule applied on (1), the following equation is obtained :

$$
\tau_{i}=\rho_{i} \frac{\left|\mathbf{N}_{\mathbf{i}}\right|}{|\mathbf{M}|}
$$

We are now looking for the regions where $\tau \leq \tau_{\max }$ called the force workspace.

\subsection{Border of the force workspace}

By its definition, the border of the force workspace is defined by points where $|\tau|=\tau_{\max }$. 
- First, suppose that $|M| \neq 0$

- If $\tau_{i}=\tau_{\max }$ then (2) can be rewritten as

$$
\tau_{\max }|M|-\rho_{i}\left|N_{i}\right|=0
$$

The curves associated to these equations are called the $n$-type curves.

- If $\tau_{i}=-\tau_{\max }$ then (2) can be rewritten as

$$
\tau_{\text {max }}|M|+\rho_{i}\left|N_{i}\right|=0
$$

The curves associated to these equations are called the $m$-type curves.

It can be noticed that the $n$ and $m$-type curves are not algebraic.

- Now, suppose that $|M|=0$ The only case with a physical sense is when all the determinent $\left|N_{i}\right|=0$. The points where the determinent of both matrices $M$ and $N_{i}$ are equal to zero are labelled as the points $N$. We have to remark that the points $N$ are some singular points which belong to the $n$ and $m$-type curves.

After this short mathematical study, it can be affirmed that the border of the force workspace is defined by portion of non algebraic curves of type $n$ and $m$, with eventually some singular points $N$.

\section{Algorithm to compute the force workspace border}

This section presents the principle of an algorithm which allows us to obtain the area defined by the relation $|\tau| \leq \tau_{\max }$. The presented scheme was inspired by a previous work on the workspace of planar parallel manipulator [].

- step 1 : key points computation

The intersections between $n$ and $m$-type curves, and the $N$ points constitute the set of key points.

Remark we can use the equations

$$
\tau_{\text {max }}^{2}|M|^{2}-\rho_{i}^{2}\left|N_{i}\right|^{2}=0
$$

to represent the curves, called q-type curves. These contain the $n$ and $m$-type curves and have the interesting property to be algebraic. That is why these equations are used to find the key points. 
Actually, the algebraic nature permits the use of the resultant []of two q-type equations and to obtain an univariate polynomial. So we just have to solve this polynomial and attribute the key point to the corresponding curves in the corresponding list of key points. The $N$ points can be computed following the same method.

- step 2 : plotting the curves

The curves are plotted with the help of the key points previously computed. The procedure which details this task will be explained later in section[]. It can be remarked that a portion of the curve between two key points is either entirely part of the force workspace border or entirely not a part of the border.

\section{- step 3 :}

To determine wether or not such a portion of a curve belongs to the force workspace border, one needs to select any arbitrary point $M$ of this curve and then computes the two normals on this point $\mathbf{v} \mathbf{1}$, $\mathbf{v} 2=-\mathbf{v} \mathbf{1}$ as well as the derivatives $u_{1}$ and $u_{2}$ of $\tau_{i}$ with respect to the position variables.

If $u_{1} v_{i}[1]>0$ and $u_{2} v_{i}[2]>0$ (A) then any point which is in the neighourhood of $M$ following the same direction as $v_{i}$ have its internal joint forces in the structure which have a value greater than $\tau_{\max }$ for a $n$-type curve and a value greater than $-\tau_{\max }$ for a m-type curve.

Reciprocally, if $u_{1} v_{i}[1]<0$ and $u_{2} v_{i}[2]<0$ (B) then any point which is in the neighourhood of $M$ following the same direction as $v_{i}$ have internal joint forces in the structure which have a value lower than $\tau_{\max }$ for a $n$-type curve and a value lower than $-\tau_{\max }$ for a m-type curve.

Consequently, if one normal $v_{i}$ follows the property (A) and the other satisfies the property (B), it can be affirmed that the point $M$ belongs to the curve. As previouslymentioned, a portion of the curve between two key points is either entirely part of the force workspace border or entirely not a part of the border. So the portion of the curve which $M$ belongs to determines a part of this border.

\subsection{Procedure to plot curves}

First of all, suppose the curves are plotted in a given domain

- Starting with one of the key point $P_{j}=\left(x_{j}, y_{j}\right)$ which is on a curve described by the equation $f(x, y)=0)$. 
- Then the tangents $T_{1}$ and $T_{2}=-T_{1}$ of the curve are computed on this initial key point.

- For each of these two tangents

- the nearest key point $P_{k}$ to $P_{j}$ is looked for.

- If $T_{i}[1]^{2} \geq T_{i}[2]^{2}$ then we define $X=x_{j}+\Delta T_{i}[1], Y=y_{j}+\epsilon$ where $\Delta$ is an incremen given by the user. The equation $f(X, Y)=f(\epsilon)=0$ can then be solved by taking an interval centered on 0 for $\epsilon$ (typically [-0.1,0.1]). If the equation $f(\epsilon)=0$ admits only one solution then the new point $\mathrm{M}$ whose coordinates are $\left(x_{j}+\Delta T_{i}[1], y_{j}+\epsilon\right)$ belongs to the studied curve. As it was said previously this point is on the force workspace border or is not.

- If $T_{i}[2]^{2} \geq T_{i}[1]^{2}$ we pose $X=x_{j}+\epsilon, Y=y_{j}+\Delta T_{i}[2]$ and the same approach is followed. If there is only only one solution, the new point $M$ whose coordinates are $\left(x_{j}+\epsilon, y_{j}+\Delta T_{i}[2]\right)$ belongs to the studied curve.

- This process is repeated continuously. If the point $M$ is near to the initial key point $P_{j}$ or its nearest key point $P_{k}$, the value of $\Delta$ is eventually adjusted to obtain as the new point $M=P_{j}$ or $M=P_{k}$.

- If the tangents $T_{1}, T_{2}$ computed on the point $M$ didn't respect the original condition obtained on $P_{j}$, the point $M$ is stored as a new key point and then we start with the next key point.

- If point $M$ coincide exactly with $P_{j}$ or one of its coordinates is outside the studied domain, we start with the next key point.

After this process, all the portion of curves beween two key points are determined, as it was said previously []. One way to increase the efficiency of the presented algorithm is to test wether or not a curve portion belongs to the force workspace boundary as soon as a point $M$ is computed. Once it is foud that a point $M$ does not belong to the boundary, there is no interest to continue the plotting of this portion.

\section{Results}

The algorithm described in the paper was tested for the for the well known planar parallel robot $3-R \underline{P} R$ [].As previously mentioned, the presented scheme provides the force workspace or all the curves involved in the determination of the area defined by $|\tau| \leq \tau_{\max }$. 


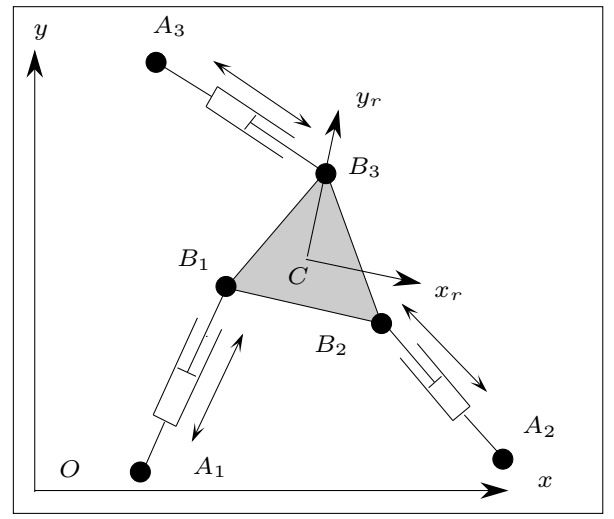

Figure 1. $3-R \underline{P} R$

All the curves presented are plotted using these input values. The coordinates of the points $A_{i}$ are given in the reference frame, and those of the points $B_{i}$ in the mobile frame.

$$
\begin{array}{lr}
O A_{1}=(0,0) & C B_{r 1}=(-4,4) \\
O A_{2}=(20,0) & C B_{r 2}=(4,-4) \\
O A_{3}=(12,10) & C B_{r 3}=(0,2)
\end{array}
$$

The threshold $\tau_{\max }=3$ and the incriminent $\Delta=0.01$ for all the results presented. Recall that our algorithm provides some results only if the orientation variable $t$ and the wrench exerced on the environnement $\mathcal{F}$ are given.

Here some results with $t=0.1$ radians are given
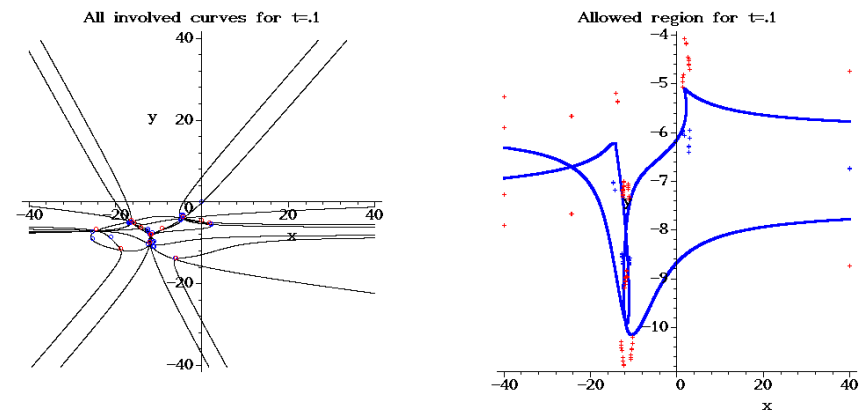

Figure 2. results with $\mathcal{F}=(4,0,0)$ 
Another results with $t=-0.1$ radians
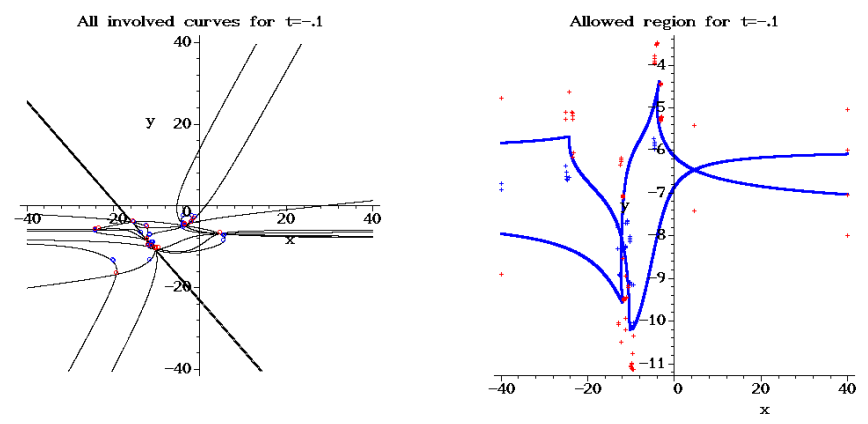

Figure 3. results with $\mathcal{F}=(4,0,0)$

Other results with various $t$ and $\mathcal{F}$.
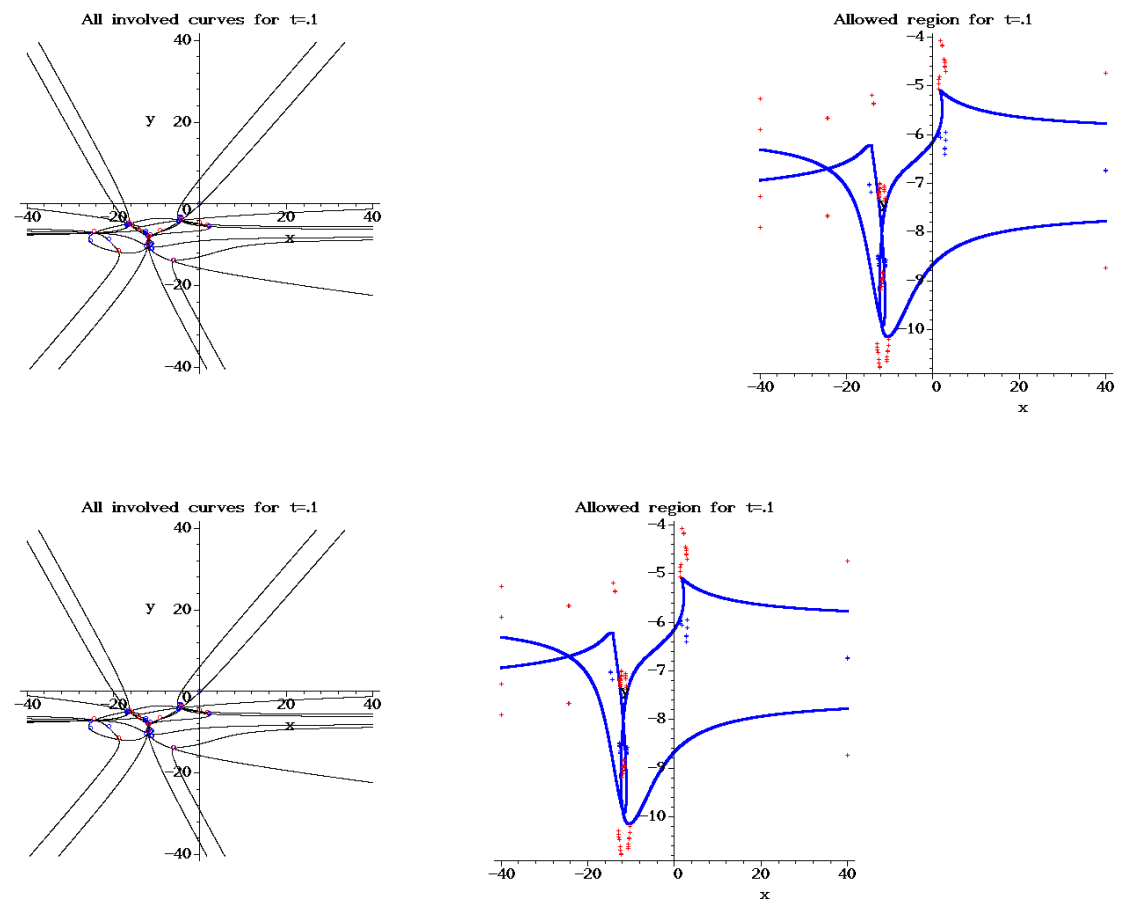

Figure 4. 


\section{Conclusion}

We have presented in this paper a method which enables to determine the force workspace of a planar manipulator for a given orientation. Although the modelisation of the $3-R \underline{P} R$ doesn't take into account the uncertainties, it may be useful to build some hybrid method using interval analysis by reducing the size of the domain.

The article also extend the singularity concept to an area around the points whose Jacobian matrix determinent is equal o zero. The metric used to build these regions has a physical sens that can be used by designers of planar parallel robots.

\section{Referencing}

The reference list should be in an alphabetical order. The provided class file will produce the format you see, which is correct. A citation in the paper should have the form Firstauthor et al., 1955, Arkauthor, 1988, Anotherauthor and Onemore, 2001. You must use the square bracket in \bibitem[Lenarcic, 1999] \{len\} in order to have Lenarcic, 1999 appear when you use \cite\{len\}.

\section{Acknowledgements}

The research work reported here was made possible by Grant No. 0000 of ARKansas Research Council.

\section{References}

Anotherauthor, C., and Onemore, F.D. (2001), This is a conference paper, Proceedings of the 1st Conference, New York, USA.

Arkauthor, A.B. (1988), My first book title, Bologna, The Famous ARK Press Company.

Firstauthor, A., Secondauthor, B., and Thirdauthor, C. (1955), This is the most famous journal paper, ASME Journal of Applied Mechanics, vol. 77, no. 11, pp. 215221. 